\title{
Analyzing Lithium Ion Battery Materials with Valence EELS
}

Fernando C. Castro ${ }^{1}$, Vinayak P. Dravid ${ }^{1,2}$.

1. Department of Materials Science and Engineering, Northwestern University, Evanston, IL, USA

2. NUANCE Center, Northwestern University, Evanston, IL, USA

Many key structure-property relationships important to lithium ion battery (LIB) materials are revealed only at the nanoscale and below. Electron Energy Loss Spectroscopy (EELS) in the Scanning Transmission Electron Microscope (STEM) is an excellent tool for studying both static and dynamic phenomena in battery materials as the combination enables elemental characterization with spatial resolution down to the atomic scale. EELS is also one of the best methods of analyzing light elements like lithium and oxygen, which are of critical importance to LIB materials.

Routine EELS analysis of LIB materials typically examines the fine structure of lithium and oxygen edges, in addition to peak intensity ratios of the transition metal white-lines. Recently, studies used the low-loss valence EELS region $(2 \mathrm{eV}-30 \mathrm{eV})$ of battery materials to visualize composition evolution. For example, an interband transition feature at $\sim 6 \mathrm{eV}$ was used to image the delithiation of $\mathrm{LiFePO}_{4} \mathrm{With}$ energy filtered TEM [1]. In-depth analysis of the plasmon region revealed the different compounds found in the solid electrolyte interface formed on Si after electrochemical cycling [2].

Here, we discuss the valence EELS structure of several LIB materials of interest, primarily using $\mathrm{LiCoO}_{2}$ as a model system. EELS spectra were collected using a Hitachi HD2300 STEM, equipped with a FEG source operating at $200 \mathrm{kV}$. The typical energy resolution during acquisition was $0.8 \mathrm{eV}$, based on the full-width at half-maximum of the EELS zero-loss peak.

The valence EELS fine structures of several LIB materials are highlighted in Figure 1. Although the $\mathrm{LiFePO} / \mathrm{FePO}_{4}$ couple has the strongest feature differences, each material still has a distinct fine structure that can be used for analysis. Furthermore, comparing the valence EELS features of $\mathrm{LiCoO}_{2}$ to its $\mathrm{Li}-\mathrm{K}$ edge shows that the valence EELS features possess higher jump ratios than the Li-K edge. This holds true especially when minimal acquisition times are used, or the spectra are collected from regions with thicknesses greater than two inelastic mean free paths.

EELS spectrum image results using valence EELS ( $7 \mathrm{eV}$ feature) of $\mathrm{LiCoO}_{2}$ can also have some advantages over mapping the Li-K edge intensity. Both types of EELS maps highlight the thin edge of the $\mathrm{LiCoO}_{2}$ region but have darker contrast in the thicker regions due to lower signal. However, calculating the $2^{\text {nd }}$ derivative of the $\mathrm{LiCoO}_{2}$ EELS spectrum isolates the $7 \mathrm{eV} \mathrm{LiCoO}$ feature from other valence EELS contributions. This processing also reduces changes in contrast due to lower signal in thick regions. As a result, the $2^{\text {nd }}$ derivative of the valence EELS region produces a map, as seen in Figure 2, that more accurately reflects the morphology and distribution of $\mathrm{LiCoO}_{2}$ particles compared to the Li-K edge, especially in areas where the thickness is greater than two inelastic mean free paths.

Valence EELS analysis also provides an alternative EELS signature when there is strong overlap between the Li-K edge and other low-loss signatures found in LIB materials. This flexibility, combined with their interpretability in low electron dosage regimes, or large effective sample thicknesses, makes valence EELS analysis an excellent supplementary technique to standard EELS characterization of LIBs. 
References:

[1] M. E. Holtz et al., Nano Letters 14 (2014), p. 1453-1459.

[2] M. Boniface et al., Nano Letters 16 (2016), p. 7381-7388.

[3] The authors acknowledge Dr. Eungje Lee for providing standard samples used in this work. This work was supported as a part of the Center for Electrochemical Energy Science, an Energy Frontier Research Center funded by the US Department of Energy, Office of Science, Basic Energy Sciences under award number DE-AC02-06CH11. This work made use of the EPIC facility of Northwestern University's NUANCE Center, which has received support from the Soft and Hybrid Nanotechnology Experimental (SHyNE) Resource (NSF ECCS-1542205); the MRSEC program (NSF DMR-1121262) at the Materials Research Center; the International Institute for Nanotechnology (IIN); the Keck Foundation; and the State of Illinois, through the IIN.

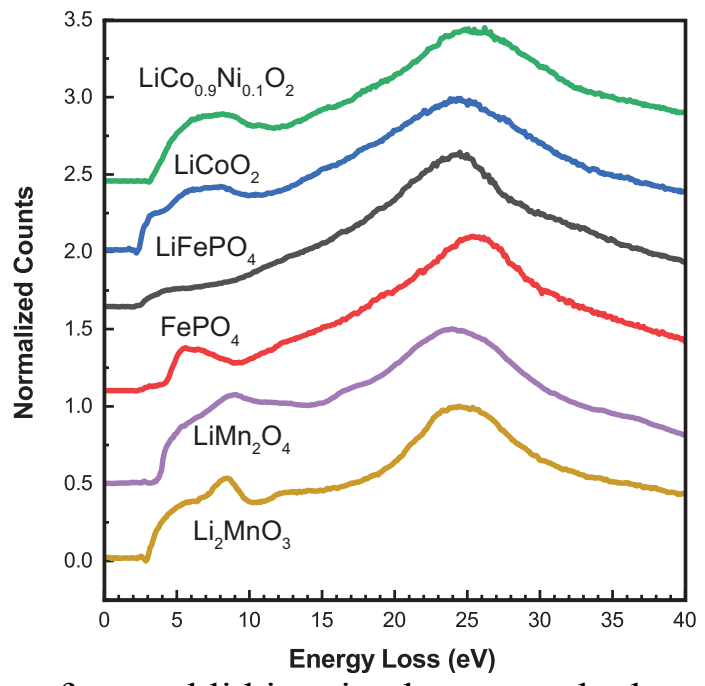

Figure 1. Valence EELS region of several lithium ion battery cathode materials.
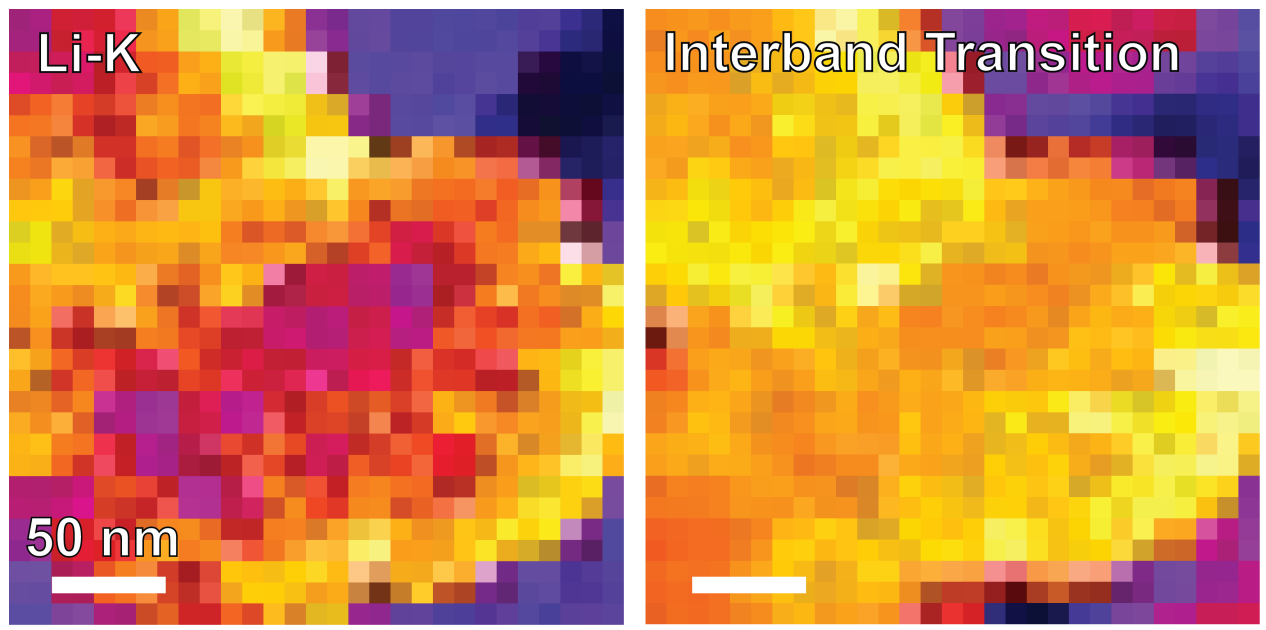

Figure 2. EELS spectrum images of the $\mathrm{Li}-\mathrm{K}$ edge and $7 \mathrm{eV}$ interband transition region of $\mathrm{LiCoO}_{2}$, after taking the $2^{\text {nd }}$ derivative of the spectra. Scale bar in both images is $50 \mathrm{~nm}$. 